\title{
Vibration tests in CFRP plates for damage detection via non-parametric signal analysis
}

\author{
P. Moreno-García ${ }^{\mathrm{a}, *}$, E. Castro ${ }^{\mathrm{b}}$, L. Romo-Melo ${ }^{\mathrm{a}}$, A. Gallego a and A. Roldán ${ }^{\mathrm{c}}$ \\ ${ }^{a}$ Applied Physics Department, University of Granada, Granada, Spain \\ ${ }^{\mathrm{b}}$ Applied Physics Department, Universidad Politécnica de Cartagena, Cartagena, Murcia, Spain \\ ${ }^{\mathrm{c}}$ Electronics and Computer Technology Department, University of Granada, Granada, Spain
}

\begin{abstract}
In this paper, two procedures for damage detection in Carbon Fiber-Reinforced Polymer (CFRP) plates are shown. One is based on the use of statistical frequency series to detect the presence of damage. This method was tested experimentally on a CFRP plate with induced damage. The other one is a procedure based on Ritz method and wavelet transform that was applied to the numerical vibration modes of a damaged CFRP plate. The former method can also be used experimentally, even if the material engineering constants are unknown.
\end{abstract}

Keywords: CFRP plates, SHM, damage detection, vibration test, Ritz method, wavelet analysis

\section{Introduction}

Damage detection in composite materials is a very interesting subject in aeronautical engineering, since it permits important maintenance cost reductions and moreover reduces the risk of critical structural failure which might produce fatal consequences in terms of human lives. Clearly it is necessary to develop new techniques to detect damage efficiently. Even if these new techniques cannot locate the damage, they can be used in conjunction with traditional ones for a complete damage characterization. Many of the new damage detection methods are based on vibration test techniques, given the fact that damage modifies the physical characteristics of the material. The natural frequencies and vibration modes therefore change with respect to the undamaged material.

The present study is divided into two parts. First, an experimental study of undamaged and damaged clamped plates is performed, and a damage index based on statistical and frequency series is defined. The damage index is an indicator capable of revealing the presence and extent of plate damage. It cannot, however, reveal its position. The piezoelectrics used in the experiments are very thin, and can thus be used as embeded sensors for Structural Health Monitoring (SHM).

Second, using the plate vibration modes obtained with a Finite Element Model (FEM), a damage location technique based on the variational Ritz method and wavelet analysis is developed. In this respect, the FEM allows us to simulate different damage types. With the Ritz method, the transversal displacement is approximated by a product series of functions compatible with the boundary conditions, as in the classical work [1].

The application of the Ritz method to damaged plates has been developed in several other works (see e.g. [2-5]). Meanwhile, wavelet analysis has been widely used to detect damage in plates [6]. In this work, one of the damage indices was taken from Ref. [7], but without using undamaged plate information as in Ref. [8].

\footnotetext{
*Corresponding author: P. Moreno-García, Applied Physics Department, University of Granada, 18071, Granada, Spain. E-mail: pmorenogarcia@ugr.es.
} 
The two methods proposed in this paper were applied to experimental data and simulated data, respectively. The first one was applied to time signals measured at particular points of the plate, while the second was applied to the plate modes, which have a spatial distribution along the plate: this may convey an interesting global idea of the differences between the two methodologies (local and global).

\section{Experimental vibration tests: Damage evaluation using frequency response functions}

\subsection{Experiment description}

The experimental part is based on the detection of damage present in a CFRP plate, whose properties are described in Table 1 . The plate was excited by a random vibration signal with frequencies from 0 to $1600 \mathrm{~Hz}$ by means of a piezoelectric actuator. The response signal of the plate was measured with four piezoelectric sensors positioned symmetrically with respect to the center. Table 2 shows the properties and position of the piezoelectric sensors. The four sides of the plate were clamped. To do so, the plate was fixed with a rigid frame and the screws were tightened with a $60 \mathrm{Nm}$ torque. Piezoelectric sensors were connected with STP cables to the Brüel and Kjær PULSE input channels in order to cancel out electromagnetic interference (EMI) and crosstalk between neighboring pairs and external sources. The input signal was also generated with the PULSE output port. The Piezosystem EPA-104 amplifier boosted, with $\times 20$ gain, the PULSE output signal to the actuator. To minimize the EMI and electrical noise, all equipment, connection boxes, cable shields and the frame were connected to an independent ground system. To reduce the influence of external vibrations, the frame was fixed to an anti-vibration table and the measurements were carried out at night with minimal ambient noise. Figure 1 shows the plate in the experimental situation with M1 (described below) attached and the whole connection diagram of the experiment.

Sixteen undamaged signals were considered in order to ensure a good statistical representation of the baseline structure, i.e. the undamaged structure. To this end the mean of the sixteen FRFs in each sensor location, with a different input signal for each (i.e., a different seed for white random signal generation) was considered as the baseline. Each set was repeated using a centered actuator position, A1, and then an offset one, A2.

Table 1

Properties of the CFRP plate

\begin{tabular}{cc}
\hline Side $(a)$ length & $85 \mathrm{~cm}$ \\
Side $(b)$ length & $65 \mathrm{~cm}$ \\
Longitudinal Young's moduli $\left(E_{x}\right)$ & $1.50 \times 10^{11} \mathrm{~Pa}$ \\
Transversal Young's moduli $\left(E_{y}\right)$ & $1.15 \times 10^{10} \mathrm{~Pa}$ \\
Poisson's ratio $(v)$ & 0.42 \\
Shear moduli $(G)$ & $5.0 \times 10^{9} \mathrm{~Pa}$ \\
Density $(\rho)$ & $1560 \mathrm{~kg} / \mathrm{m}^{3}$ \\
Layer thickness $(h)$ & $0.188 \mathrm{~mm}$ \\
Number of layers & 12 \\
Stacking sequence & {$[0 / 90]_{3 \mathrm{~s}}$} \\
\hline
\end{tabular}

Table 2

Properties and position of piezoelectrics.

\begin{tabular}{ccccccc}
\hline \multicolumn{1}{c}{ Use } & Ceramics & Electrode & Thickness & Diameter & Positions & Notation \\
\hline Actuator & PIC255 & $\mathrm{Ag}$ & $0.5 \mathrm{~mm}$ & $20 \mathrm{~mm}$ & $(42.5,32,5),(20,14) \mathrm{cm}$ & $\mathrm{A} 1, \mathrm{~A} 2$ \\
Sensor & PIC151 & $\mathrm{Ag}$ & $0.5 \mathrm{~mm}$ & $10 \mathrm{~mm}$ & $(24.5,21.5),(24.5,43.5)$, & $\mathrm{S} 1, \mathrm{~S} 2$, \\
& & & & & $(60.5,21.5),(60.5,43.5) \mathrm{cm}$ & $\mathrm{S} 3, \mathrm{~S} 4$ \\
\hline
\end{tabular}


The damage was simulated by masses attached to the plate with weights: $6.7 \mathrm{~g}$ (M1), $12.8 \mathrm{~g}$ (M2), $24.7 \mathrm{~g}$ (M3), $39.6 \mathrm{~g}$ (M4), $85.8 \mathrm{~g}$ (M5) and $192.9 \mathrm{~g}$ (M6), always at position $(67,49.5) \mathrm{cm}$. The experimental setup for the damaged case was the same as the undamaged, but in these experiments five measurements were made instead of sixteen. The input of each measurement was also recorded to calculate the frequency response functions (FRFs). These FRFs were computed from frequencies $200 \mathrm{~Hz}\left(f_{\min }\right)$ to $1500 \mathrm{~Hz}\left(f_{\max }\right)$. The range was selected in order to prevent possible experimental errors due to nonlinearity of the piezoelectrics or cut effects. Using FEM simulation, it was corroborated that more than 80 resonance peaks of the plate were within this range $[0-1500 \mathrm{~Hz}]$, which was considered fully sufficient for damage detection.

The attached mass type damage has the advantages of being very well controlled and nondestructive, which is especially important to validate the methods for damage detection and evaluation. Note that it is possible to do any amount of damage in an arbitrary position with any damage intensity.

\subsection{Damage detection}

In order to detect the presence of damage, the following damage index was defined and used:

$$
D_{1}=\sum_{f_{i}=f_{\min }}^{f_{\max }} \sqrt{\left|F R F_{1}-F R F_{2}\right|},
$$

where the summation is over all frequencies between $f_{\min }$ and $f_{\max }$, with a frequency resolution of $0.25 \mathrm{~Hz}$ in this case. It should be underlined that if damage produces a FRF change less than the frequency resolution, it becomes undetectable, and more resolution should be used. In the study presented here, this frequency resolution sufficed to detect all the considered damage intensities.

We started by computing $D_{1}$ for the undamaged plate. However, despite always using the same plate, there were small FRF differences in the measurements. For this reason $D_{1}$ was computed using the FRFs in each one of the sixteen undamaged measurements as $F R F_{1}$ and $F R F_{2}$. This calculation provides 120 values (the maximum number of combinations between sixteen elements) of the index for the undamaged plate. The index $D_{1}$ thus calculated was normalized to a mean average. This procedure provides a distribution of the damage index for each sensor and actuator when the plate is undamaged.

The calculation was repeated for the damaged plate, but now the average of the sixteen undamaged FRFs was used as $F R F_{1}$, and each of the new FRFs obtained from the damaged measurements was used as $F R F_{2}$. The resulting $D_{1}$ was normalized in the same way as that of the undamaged index.
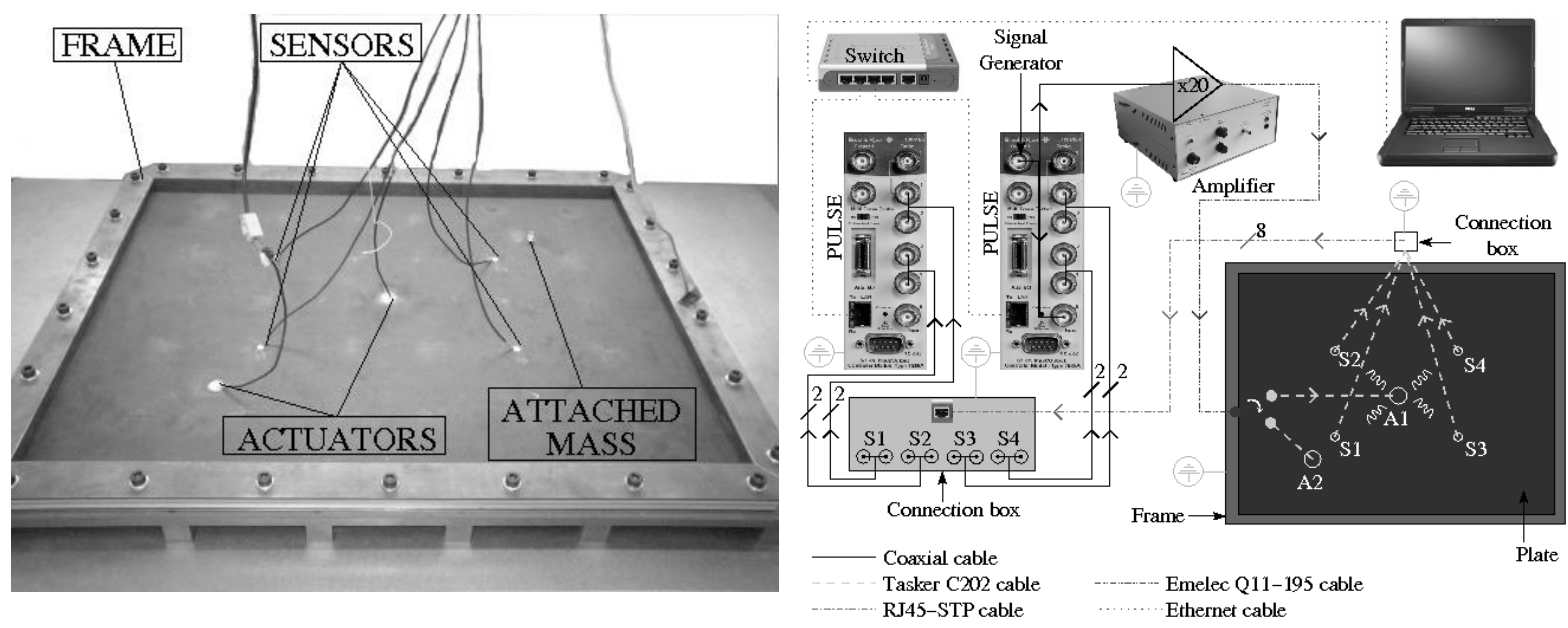

Fig. 1. Plate with mass M1 attached (left) and connection diagram of the experiment (right). 


\subsection{Results}

Figure 2 shows an example of the FRFs at the four sensors during the same measurement, using the centered actuator A1. There is evident similarity among the four signals in the frequency domain, due to the symmetric positioning of the sensors and the fact that the plate was orthotropic.

In Fig. 3, the FRFs of two different measures of the undamaged plate can be seen, along with the plates with additional mass M2 and M6 for both actuators A1 and A2. Because of the similarity of the sensors, Fig. 3 only shows the results at sensor S1. The two undamaged measures are seen to be very close, while the differences between the undamaged and added mass (particularly for M6) measures are clear in some of the peaks.

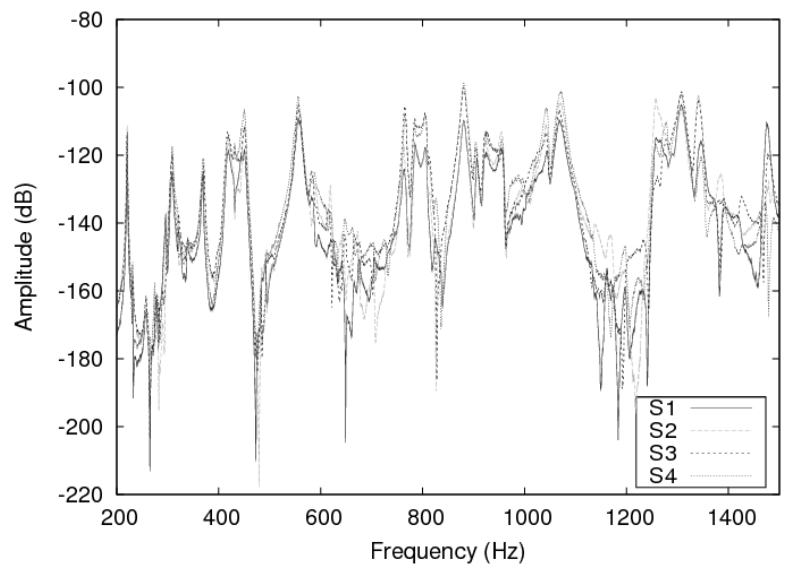

Fig. 2. FRFs at four sensors (S1, S2, S3, S4) for a particular measurement in the undamaged plate.
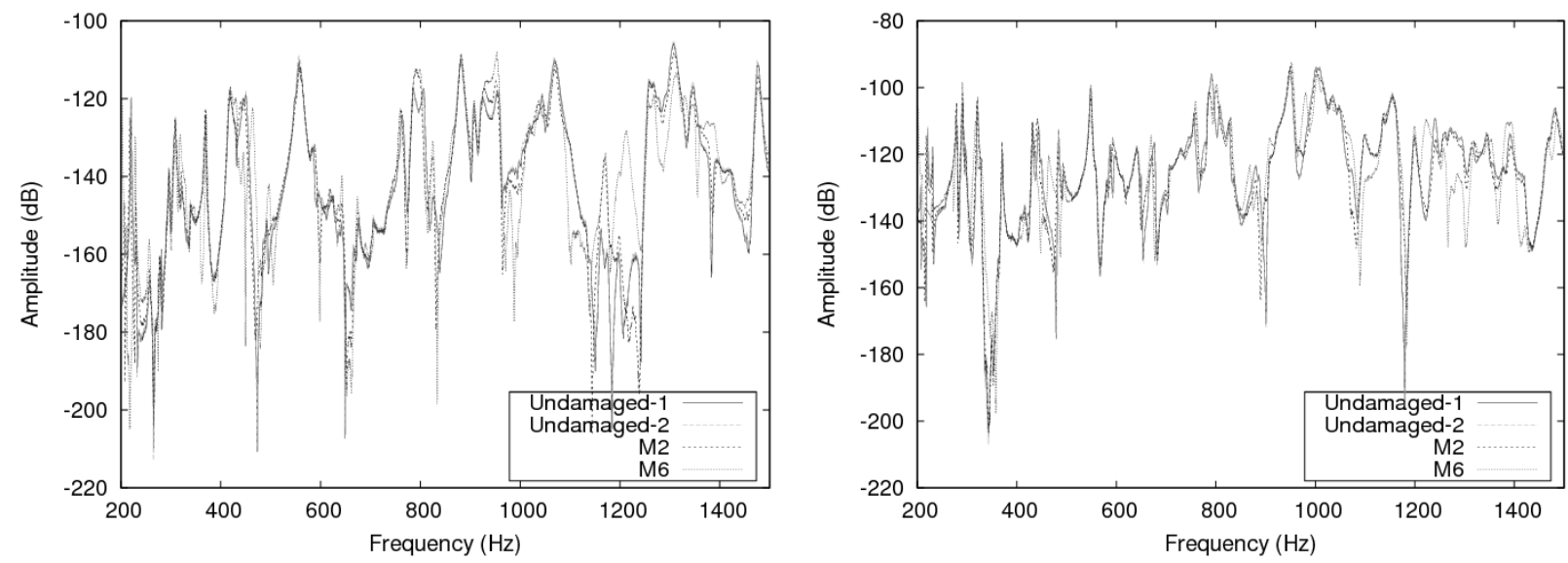

Fig. 3. FRFs for the undamaged plate and the plates with additional mass M2 and M6 at sensor S1, using the actuators A1 (left) and A2 (right).

Figure 4 shows the average of the damage index $D_{1}$ for each damage situation versus the attached mass values at the four sensors and for both actuators. Dispersion is not represented due to its small value (especially in the damaged cases), with maximum value of 0.35 for the A1-S1 combination in undamaged cases, and 0.076 for the A2-S4-M2 combination in damaged cases. It can be seen that the average $D_{1}$ values computed for the undamaged plate is equal to 1 by normalization, and differs significantly from the average $D_{1}$ values computed in a damage situation, revealing the presence of damage. Furthermore, $D_{1}$ increases when the damage increases, indicating the usefulness of $D_{1}$ as an indicator. On the other hand and in general, there is no clear relationship between $D_{1}$ and the proximity of the sensor to the damage. 

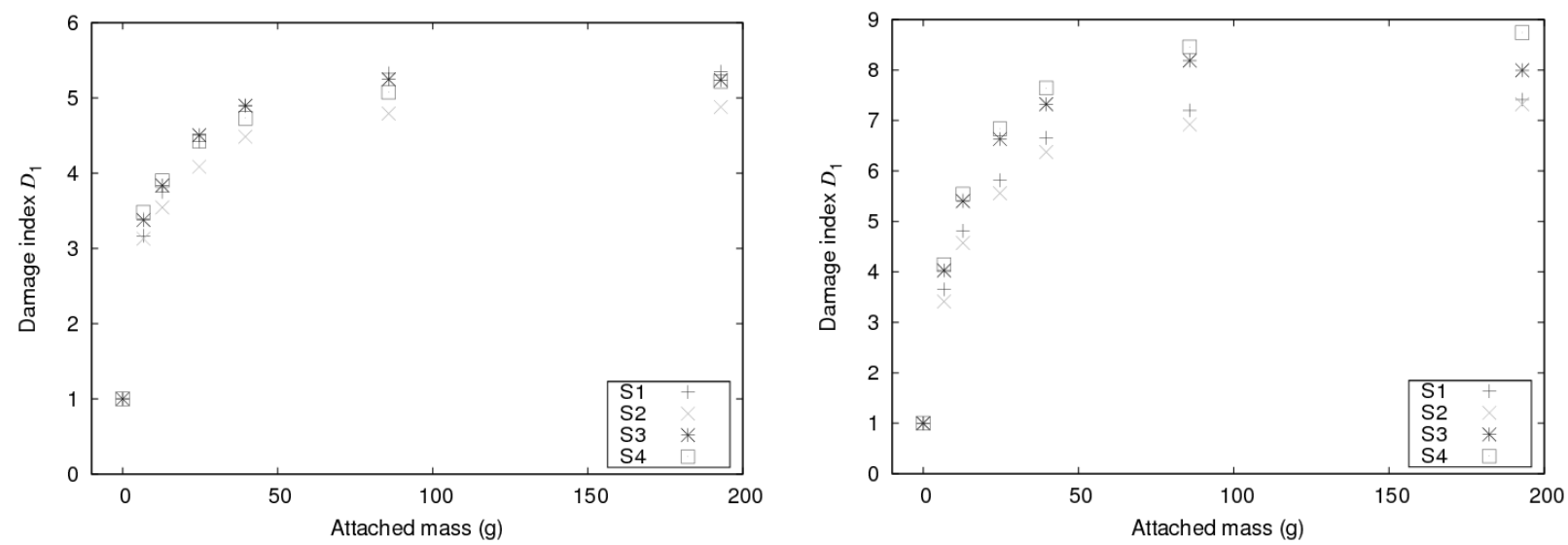

Fig. 4. Damage index $D_{1}$ for sensors S1, S2, S3 and S4 using A1 (left) and A2 (right).

\section{Numerical vibration tests: Damage location using wavelet transform}

\subsection{Semianalytical solution for the plate modes}

The Ritz method [1] is based on the calculation of plate energy. The energy $E$ can be calculated as in Ref. [2]:

$$
E=U-T,
$$

where $U$ is the potential energy and $T$ is the kinetic energy. For laminated composite materials, these energies can be expressed as

$$
\begin{aligned}
U= & \sum_{i} \frac{1}{2} \iint_{A^{i}} D_{11}^{i}\left(\frac{\partial^{2} w}{\partial x^{2}}\right)^{2}+2 D_{12}^{i} \frac{\partial^{2} w}{\partial x^{2}} \frac{\partial^{2} w}{\partial y^{2}}+D_{22}^{i}\left(\frac{\partial^{2} w}{\partial y^{2}}\right)^{2}+ \\
& +4 D_{16}^{i} \frac{\partial^{2} w}{\partial x^{2}} \frac{\partial^{2} w}{\partial x \partial y}+4 D_{26}^{i} \frac{\partial^{2} w}{\partial y^{2}} \frac{\partial^{2} w}{\partial x \partial y}+4 D_{66}^{i}\left(\frac{\partial^{2} w}{\partial x \partial y}\right)^{2} d x d y \\
T= & \sum_{i} \frac{1}{2} \iint_{A^{i}} \rho^{i} h^{i} \omega^{2} w^{2} d x d y,
\end{aligned}
$$

where $D_{k \mathrm{l}}$ are the components of the bending stiffness tensor, $w$ is the transversal displacement, $\rho$ is the density, $h$ is the thickness, $\omega$ is the angular frequency and the superscript $i$ denotes each zone of the plate with different properties (density, thickness or $D$ components). The integral is performed over the area of this zone $\left(A^{i}\right)$.

The transversal displacement can be expressed as a function product series with the corresponding function weights

$$
w(x, y)=\sum_{m=1}^{M} \sum_{n=1}^{N} A_{m n} X_{m}(x) Y_{n}(y),
$$

where $A_{m n}$ are the function weights, $M$ and $N$ are the the summation number of terms, and $X_{m}(x)$ and $Y_{n}(y)$ are functions that satisfy the boundary conditions at $x=(0, a)$ and $y=(0, b)$. In this work, the functions for a clamped-clamped beam described in Ref. [9] were used.

To obtain the vibration modes of the plate, the energy must be minimized, i.e. 


$$
\frac{\partial E}{\partial A_{m n}}=0
$$

Using Eqs (2) to (6), an eigenvalue problem is obtained, i.e.

$$
C-\lambda I=0,
$$

where $C$ is a matrix, $I$ is an identity matrix, and $\lambda$ the eigenvalues, the square of the angular frequencies. The obtained eigenvectors are in Eq. (5) the weights for each mode.

\subsection{Damage detection}

The second method proposed in this paper for damage detection is based on Wavelet Transform combined with the semi-analytical Ritz method. The method is based on the assumptiom that a damaged mode is approximately composed of an undamaged mode and other contributions related with the damage, as in Ref. [8].

Firstly, the four first (main) frequencies $\left(f_{i}\right)$ and vibration modes $\left(M_{d}\right)$ of a damaged plate are obtained using the FEM research code FEAP [10]. Then, using the summation (5) and assuming a set of boundary condition compatible functions as in Ref. [9], the eigenvectors $A_{m n}$ that fit the numerical modes are computed using a standard minimization procedure. From the eigenvectors $A_{m n}$ and the eigenvalues, the matrix $C$ of Eq. (7) is obtained. This is an approximation of the matrix that can be obtained using the Ritz method with $M=N=2$ in Eq. (5), so a standard eigenvalues algorithm is used to obtain the frequencies and vibration modes $\left(M_{a}\right)$. These new vibration modes are an approximation of the original ones, since only four terms in Eq. (5) were used. Therefore, some information is lost during the process and we can assume that this information is mainly related with the damage, so that we may use the approximation modes as the undamaged modes, as in Ref. [7].

Again, to detect the presence of damage, the following damage index was defined and used:

$$
D_{2}=\left|W\left(M_{a}\right)-W\left(M_{d}\right)\right|
$$

where $W(X)$ represents the bidimensional continuous wavelet transform (CWT) using the Halo Wavelet described in Ref. [11]. It is an isotropic wavelet with three vanishing moments, and it is very appropriate for damage detection using transversal vibration modes, as it can detect a discontinuity up to the fourth derivative of the mode. The selected CWT parameters were $k_{0}=6$ and scale equal to 16 . The considered damages were three stiffness reductions, performed via a Young's moduli reduction: $-1 \%(\mathrm{Y} 1),-5 \%(\mathrm{Y} 2)$ and $-10 \%(\mathrm{Y} 3)$. All damages consisted of a squared measuring four centimeters on each side centered at three different positions: $\mathrm{P} 1=(67,49.5) \mathrm{cm}, \mathrm{P} 2=(0.605,0.325)$ $\mathrm{cm}$ and $\mathrm{P} 3=(0.755,0.555) \mathrm{cm}$. To compute the damage index, the third mode was chosen as an example.

The main advantage of the current method is that it does not need information from the undamaged plate. Furthermore, it is worth noting that it can also be applied to experimentally measured modes instead of the FEM ones, without requiring the data of the material engineering constants. Figure 5 offers a diagram of the complete damage detection technique.

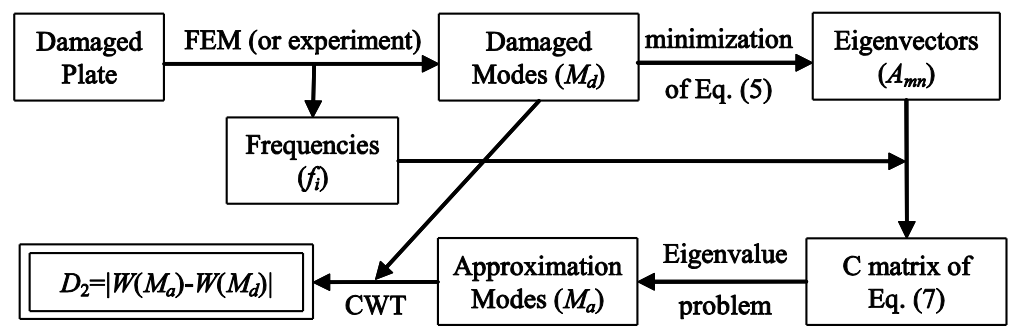

Fig. 5. Diagram for damage detection based on Ritz method and wavelet transform. 


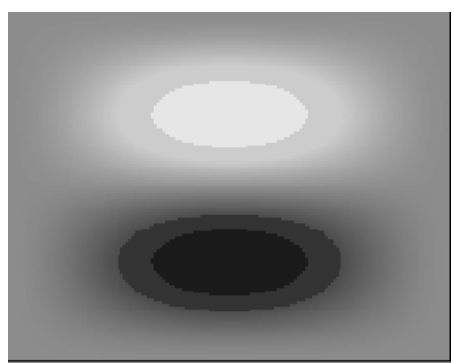

FEM Y3

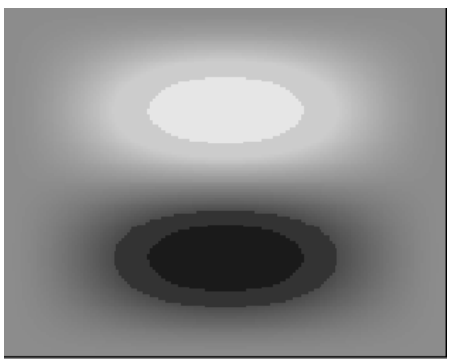

Approximation Y3

Fig. 6. Original FEM third mode (left) and approximation (right) for the Y3 damage case at position P1.

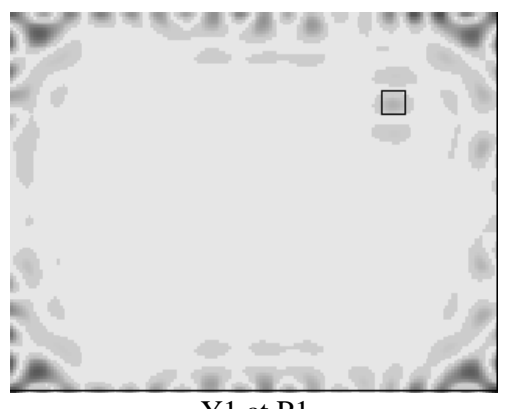

$\mathrm{Y} 1$ at $\mathrm{P} 1$

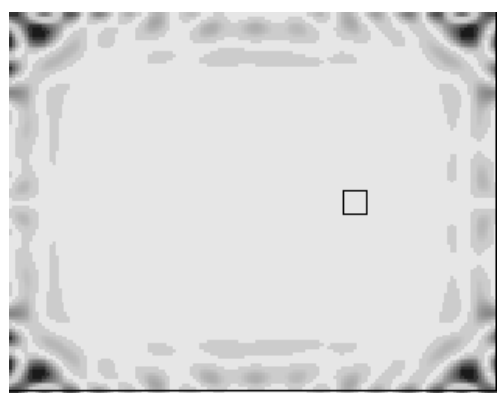

$\mathrm{Y} 1$ at $\mathrm{P} 2$

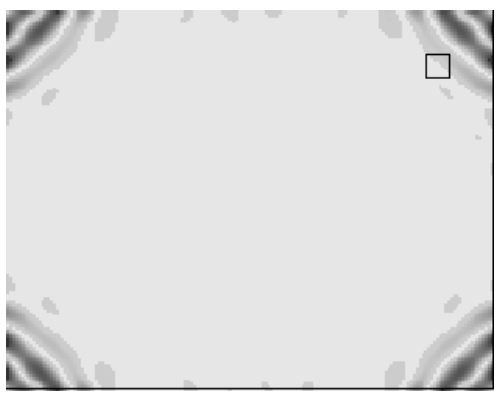

$\mathrm{Y} 1$ at $\mathrm{P} 3$

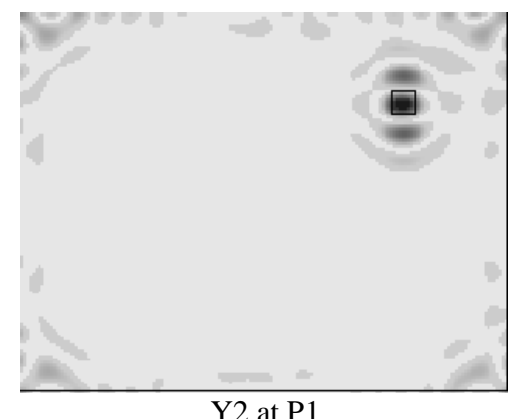

$\mathrm{Y} 2$ at $\mathrm{P} 1$

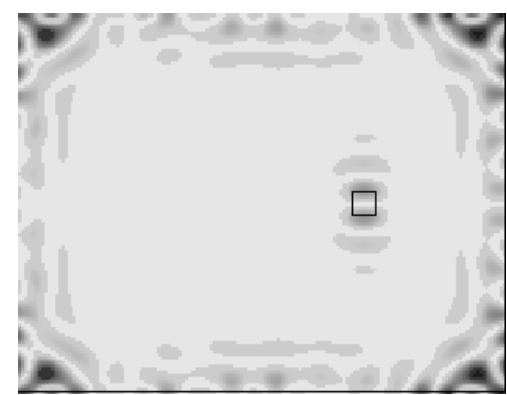

$\mathrm{Y} 2$ at $\mathrm{P} 2$

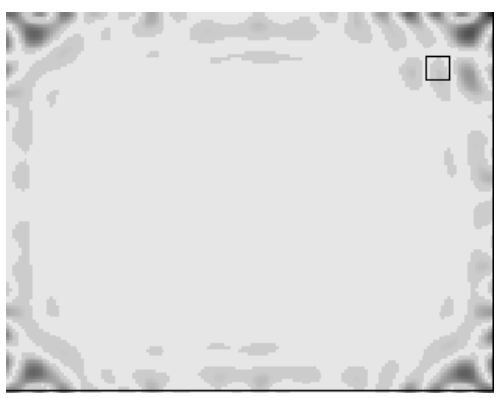

$\mathrm{Y} 2$ at $\mathrm{P} 3$

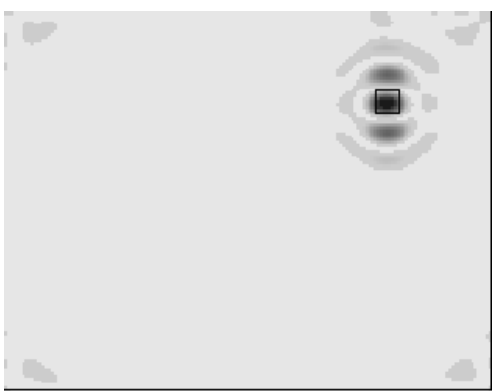

Y3 at P1

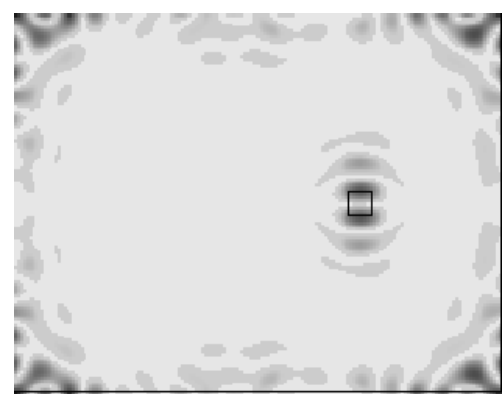

$\mathrm{Y} 3$ at $\mathrm{P} 2$

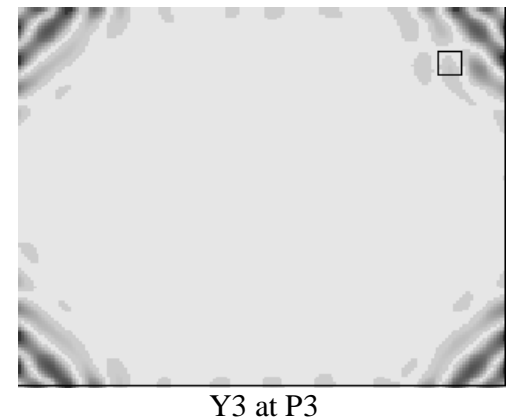

Fig. 7. Damage index $D_{2}$ for $\mathrm{Y} 1, \mathrm{Y} 2$ and $\mathrm{Y} 3$ damages at $\mathrm{P} 1, \mathrm{P} 2$ and $\mathrm{P} 3$ positions.

\subsection{Results}

Below, Fig. 6 shows the original FEM third mode (left) and the approximation (right) obtained for the Y3 damage case at position P1. Because the defect is very small, the original mode is very similar to the undamaged and it is 
impossible to perceive the damage. The approximation mode is moreover very similar to the original one.

Figure 7 displays the damage index $D_{2}$ at all plate points for Y1, Y2 and Y3 cases, at the three positions P1, P2 and P3. A square line delimits the damage extension. In most cases, it is clear that index $D_{2}$ is larger near the damage position. Additionally - and although it cannot be seen due to the different scales - the larger the damage, the larger $D_{2}$ is. Thus, $D_{2}$ is a good indicator for damage location and damage level evaluation. Some perturbations are seen by the borders. The reason is that the computed modes $M_{a}$ are an approximation of the undamaged modes, yet not exactly equal. This difference means that the boundary effects are not completely eliminated. Their presence is more evident as damage intensity decreases.

Regarding the different damage locations, Fig. 7 shows that damage index $D_{2}$ works well when the damage is located at position P1, but worse at position P2 (because the mode has lower displacement) and barely detects damage at position P3 (because it is very close to the borders).

\section{Conclusions}

In this work, two different damage indices have been defined for a CFRP plate: the first $\left(D_{1}\right)$ is applied to experimental frequency series and compares two different FRFs. The second $\left(D_{2}\right)$ is applied to vibration modes and is based on the difference of bidimensional continuous wavelet transforms of the mode, and on a new vibrational mode numerically obtained.

With respect to $D_{1}$ results, two different actuators excited the plate with a random signal, four sensors recorded the plate response and we computed the FRFs. The damage index obtained when the two FRFs were computed from an undamaged measurement differs significantly from that obtained when one of the measurements is carried out on an undamaged plate and the other one on a damaged plate. Furthermore, in general, $D_{1}$ increases as the damage increases. However, this method cannot provide information about the damage location.

To apply the second damage index $D_{2}$, vibration modes were obtained from a FEM model and eigenvectors corresponding to these modes were then obtained using a minimization procedure. From these eigenvectors and the frequencies, an approximation of the matrix $C$ of Eq. (7) was obtained. This matrix provides new modes, which are approximations of the original FEM modes, revealing the presence and location of damage given the differences between the continuous wavelet transforms of both (FEM and approximation) modes. Additionally, $D_{2}$ increases as the damage increases. On the other hand, the method does not work equally well at different damage locations, proving better when the damage is located at high displacement positions of the mode and away from the borders. This method can also be applied to experimentally measured modes, even if the material engineering constants are unknown.

\section{Acknowledgments}

The authors thank Spanish I+D National Plan "DPI2006-02970" Project and Junta de Andalucía "Proyecto de Excelencia P08-TEP-03641" for financial assistance, J. L. Pérez-Aparicio for his help with Ritz method and FEM simulations and A. Ladrón-de-Guevara and C.J. Infantes for their help solving several experimental problems.

\section{References}

[1] D. Young, Vibration of rectangular plates by the Ritz method, Journal of Applied Mechanics 17(4) (1950), 448-453.

[2] G.B. Chai, Frequency analysis of rectangular isotropic plates carrying a concentrated mass, Computers \& Structures 56(1) (1995), 39-48.

[3] D.R. Avalos, H.A. Larrondo and P.A.A. Laura, Analysis of vibrating rectangular anisotropic plates with free-edge Holes, Journal of Sound and Vibration 222(4) (1999), 691-695.

[4] P.M. Ciancio, C.A. Rossit and P.A.A. Laura, Approximate study of the free vibrations of a cantilever anisotropic plate carrying a concentrated mass, Journal of Sound and Vibration 302(3) (2007), 621-628.

[5] C.A. Rossit and P.M. Ciancio, Free vibrations of anisotropic rectangular plates with holes and attached masses, Structural Engineering and Mechanics 28(1) (2008), 53-67. 
[6] S. Loutridis, E. Douka, L.J. Hadjileontiadisc and A. Trochidis, A two-dimensional wavelet transform for detection of cracks in plates, Engineering Structures 27(9) (2005), 327-1338.

[7] E. Castro, M.T. García-Hernández and A. Gallego, Damage detection in rods by means of the wavelet analysis of vibrations: Influence of the mode order, Journal of Sound and Vibration 296 (2006), 1028-1038.

[8] H. Gökdağ and O. Kopmaz, A new damage detection approach for beam-type structures based on the combination of continuous and discrete wavelet transforms, Journal of Sound and Vibration 324(3-5) (2009), 1158-1180.

[9] J.R. Gartner and N. Olgac, Improved numerical computation of uniform beam characteristic values and characteristic functions, Journal of Sound and Vibration 84(2) (1982), 481-489.

[10] R.L. Taylor, FEAP: A Finite Element Analysis Program, http://www.ce.berkeley.edu/projects/feap/.

[11] T. Dallard and G.R. Spedding, 2-D wavelet transforms: Generalizations of Hardy space and application to experimental studies, European Journal of Mechanics B/Fluids 12(1) (1993), 107-134. 

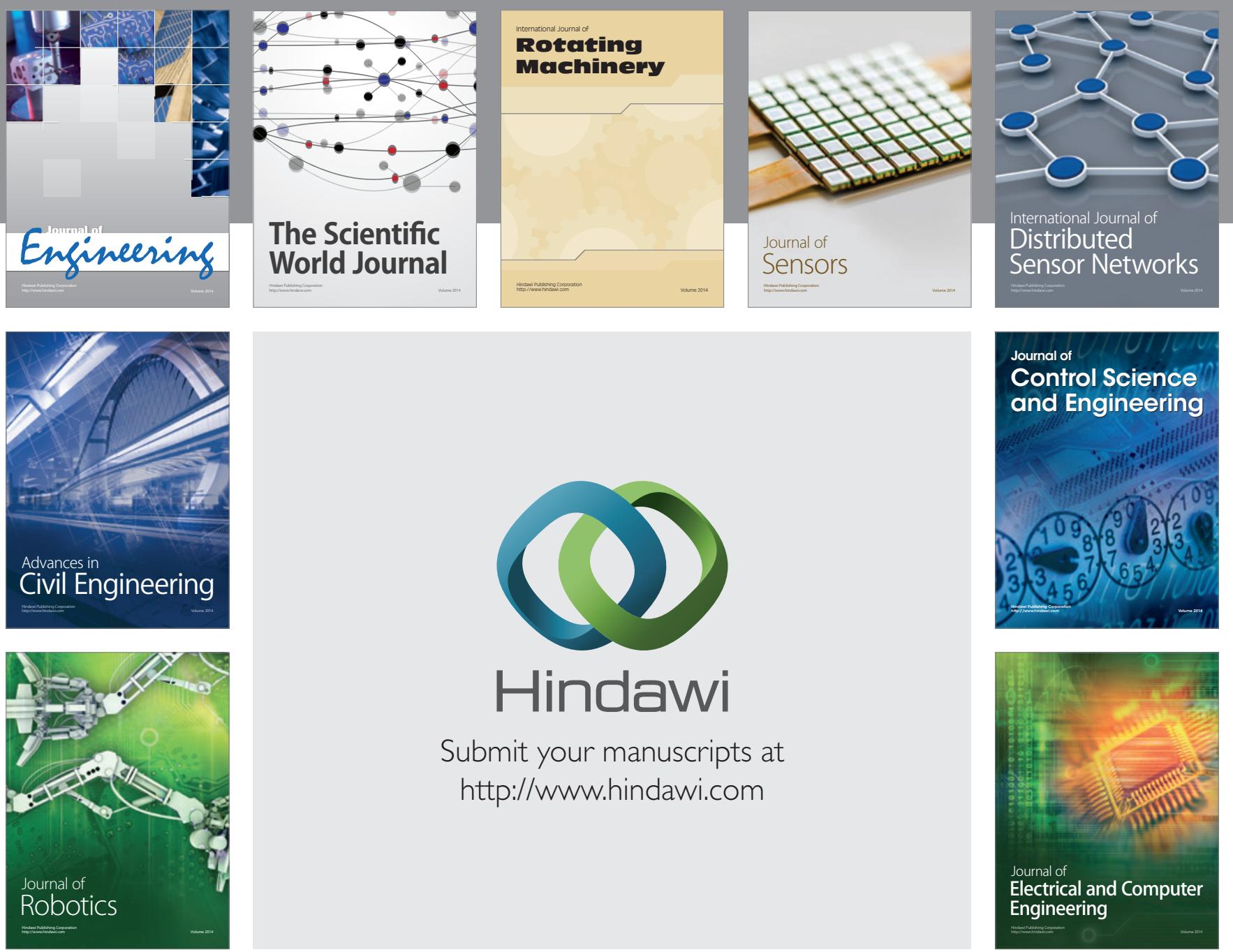

Submit your manuscripts at

http://www.hindawi.com
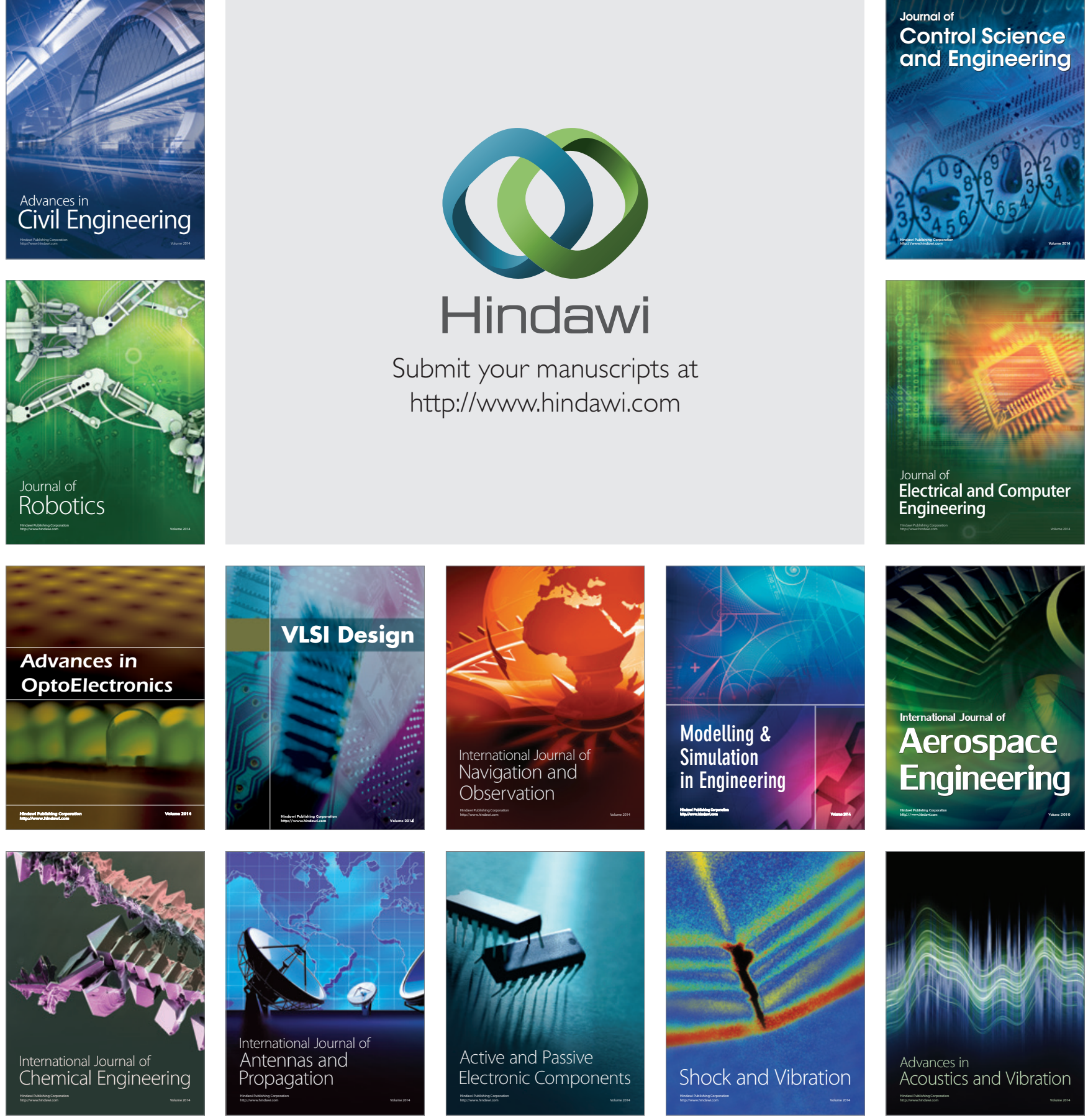